\title{
Einheitliche Regionalplanung für die Metropole Ruhr: ein Erfolgsmodell?
}

\section{Unified regional planning for the Ruhr conurbation: A model for success?}

\begin{abstract}
Kurzfassung
Die Übertragung der Regionalplanung für die „Metropole Ruhr“ auf den Regionalverband Ruhr (RVR) ist vor dem Hintergrund des zunehmenden Standortwettbewerbs als wichtiger Schritt zur stärkeren Berücksichtigung kommunenübergreifender Belange zu begrüßen. Die Regionalplanungsinstanz RVR steht hier vor der von allseitiger Akzeptanz und politischem Geschick abhängigen Gratwanderung zwischen kommunalem Eigeninteresse und den Interessen des Ruhrgebiets als Ganzes. Die Metropole Ruhr, definiert durch die momentane Gebietskulisse des RVR, hat ungleich bessere Möglichkeiten, sich im internationalen Standortwettbewerb zu behaupten, als jede einzelne Kommune für sich genommen.
\end{abstract}

\begin{abstract}
Responsibilities for regional planning in the Ruhr conurbation have now been assigned to the Ruhr Regional Association (RVR). This represents an important step towards giving greater attention to the needs of the entire region. This is an essential step, given the increasing competition among potential operating locations. $R V R$, acting as the regional planning unit, will face a balancing act between individual municipalities' own interests and the interests of the Ruhr as a whole. The success achieved by RVR will depend on its attaining universal acceptance and applying political skills. The Ruhr metropolitan area, as defined by the RVR boundaries, is far better equipped than any single town or city to maintain a viable position as potential operating sites contending internationally for investors' favour.
\end{abstract}

\section{Ist die Vision einer „Metropole Ruhr“ sinnvoll?}

\subsection{Regionalisierung des Standortwettbewerbs}

Wirtschaftliche Integration in Europa und der fortwährende Strukturwandel in den Staaten, Bundesländern und Regionen erfordern Schritt haltende Anpassungen der Verwaltungsstrukturen, wenn die öffentliche Hand ihr Leistungsangebot zum Wohl der Bürger erhalten bzw. ausbauen und gleichzeitig effizient wirtschaften soll. Dabei sind die oft vermutete höhere Effizienz zentralistischer Organisation und das Prinzip der Subsidiarität, das die Selbstverantwortung der Bürger und Kommungen stärkt und Entdeckungsprozesse in Richtung besserer Lösungen zulässt, gegeneinander abzuwägen. Es liegt nahe, dass Kommunen, die mit ähnlichen Herausforderungen konfrontiert sind, durch eine stärkere Integration mehr profitieren können als andere. Darüber hinaus werden sich in Zukunft nur solche Regionen im Standortwettbewerb als Kristallisationspunkte wirtschaftlicher Aktivität durchsetzen können, die in ihrem Auftritt nach außen über eine hinreichende "kritische Masse“ verfügen.

Vor einer derartigen Anpassung steht derzeit auch das Ruhrgebiet. ${ }^{1}$ Die vom Landtag Nordrhein-Westfalen beschlossene Übertragung der Regionalplanung für die Metropole Ruhr auf den Regionalverband Ruhr (RVR) ist vor dem Hintergrund des zunehmenden Standortwettbewerbs als wichtiger Schritt zur stärkeren Berücksichtigung kommunenübergreifender Belange im 


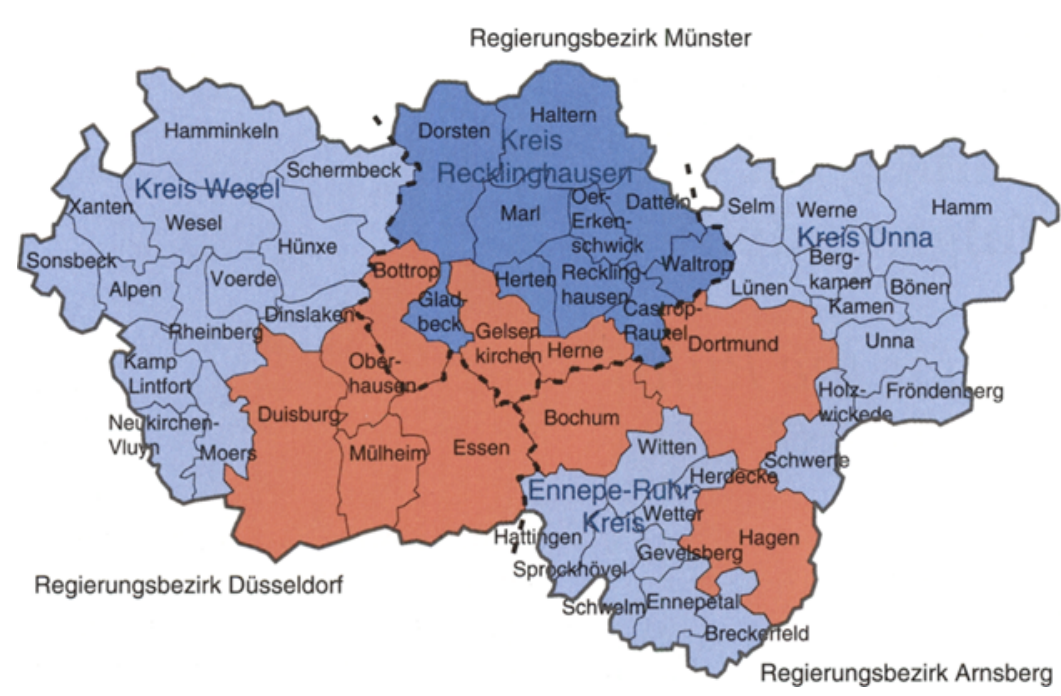

Abbildung 1

Kreise, kreisfreie Städte und Gemeinden des Ruhrgebiets
Ruhrgebiet zu begrüßen. ${ }^{2}$ Sie gibt - ohne an der historisch geprägten Aufteilung der Regierungsbezirke etwas zu ändern - den Selbstverwaltungsorganen des Ruhrgebiets ein Stück regional-integrativer Gestaltungskompetenz zurück, das diese in ähnlicher Form schon einmal, nämlich bis 1975 besessen hatten. Die Übertragung kann ihren Zweck allerdings erst dann voll erfüllen, wenn die planende Institution auch mit entsprechenden finanziellen und personellen Ressourcen ausgestattet wird sowie die Entscheidungshoheit über die zur Umsetzung der Regionalplanung nötigen Finanzmittel erhält. Angesichts des Gebots eines sparsamen Umgangs mit Steuermitteln müsste dabei die Ressourcenausstattung untergeordneter Verwaltungsebenen Zug um Zug zurückgefahren werden. Auf längere Sicht sollte zudem über weitergehende Formen einer administrativen Integration des Agglomerationsraums an Rhein und Ruhr nachgedacht werden.

Das Ruhrgebiet hat aufgrund seiner Bevölkerungszahl, Siedlungsstruktur und wirtschaftlichen Potenziale das Zeug, in Zukunft eine weltweit als solche wahrgenommene "Metropole Ruhr" zu werden. Heute stellt sich das Gebiet des Regionalverbands Ruhrgebiet (Abb. 1) allerdings eher als große städtische Agglomeration dar, die trotz wesentlicher Gemeinsamkeiten im wirtschaftlichen, sozialen und kulturellen Bereich für Außenstehende nicht als Einheit erkennbar ist und deren Interessen im Hinblick auf die Regionalplanung aufgrund der Zuständigkeit dreier Regierungspräsidien momentan noch nicht einheitlich vertreten werden. Es wird sich aber im 21. Jahrhundert nur dann zu einer mit London, Paris oder Berlin vergleichbaren „Metropole" entwickeln können, wenn es einem einheitlichen, demokratisch in der Region basierten politischen Gestaltungswillen unterworfen wird, was erheblich mehr als eine einheitliche Regionalplanungskompetenz erfordert. Schließlich gilt es, bei der Stärkung von Wirtschaftsbereichen und der Verbesserung der für Wirtschaft und Lebensqualität relevanten Infrastrukturen gewisse Schwerpunkte zu setzen. Hiervon können die Attraktivität der Region insgesamt und damit auch ihre wirtschaftliche Entwicklung profitieren.

Der Strukturwandel lässt sich durch Regionalplanung freilich nicht im strengen Sinn des Wortes "steuern". Er ist vielmehr primär von den Allokationsentscheidungen der Privaten abhängig, die dabei zwar die positiven oder negativen Anreize berücksichtigen, welche die Planungsinstrumente setzen, aber dennoch weitgehend autonom handeln. Für einen "großen Wurf" bedürfte es zudem eines weit kühneren Integrationskonzepts, das angesichts der in allen politischen Lagern verankerten Widerstände gegen eine stärkere Integration zumindest augenblicklich nicht in Sicht scheint.

\subsection{Das Konzept der regionalen Wettbewerbsfähigkeit}

Folgt man der Diskussion in der regionalökonomischen Forschung, überwiegen die Argumente dafür, das Gewicht des Agglomerationsraums "Metropole Ruhr" im Standortwettbewerb durch eine Regionalplanung zu stärken, die die bestmögliche Allokation der innerhalb des Ruhrgebiets verfügbaren Flächen auf geeignete Nutzungen unterstützt. Zwar sind aus raumökonomischer Sicht die Ursachen der ungleichen Verteilung der Ressourcen im Raum und das langfristige Verhältnis von auf Konvergenz und auf Divergenz drängenden Kräften (hierzu z. B. die Analysen in Fujita/Krugman/ Venables 2001) keineswegs abschließend geklärt. Die empirische Regionalforschung hat ungeachtet einer 
noch ausstehenden Klärung dieser grundsätzlichen Fragen aufgezeigt, dass in der wirtschaftsräumlichen Entwicklung zunächst erhebliche Ungleichheiten zwischen den Regionen auftreten. In den 1950er Jahren wurden wegen diesen Beobachtungen sog. Polarisationsansätze formuliert, die davon ausgehen, dass die Wirtschaftsentwicklung durch „motorische Einheiten" vorangetrieben wird. Dabei handelt es sich um Branchenschwerpunkte, die in der Regel eine regionale Standortkonzentration aufweisen (Perroux 1950; Hirschmann 1958; Myrdal 1957). Die Entstehung und Ausbreitung von Neuerungen - seit Schumpeter als Triebfeder der Wirtschaftsentwicklung bekannt - wird seitdem auch als Ursache für regionale Disparitäten und deren Verfestigung angesehen. Ende des 20. Jahrhunderts wurden polarisationstheoretische Elemente im Rahmen der endogenen Wachstumstheorie (Romer 1990) und der „New Economic Geography“ (Krugman 1991) in das formale Gerüst der neoklassischen Ökonomik integriert. Viele Autoren haben seit dieser Zeit die Bedeutung der Innovationsstärke ansässiger Unternehmen für die Wettbewerbsfähigkeit von Regionen aufgezeigt (Malecki 1991; Camagni 2002).

Auch Porter $(1990,1998)$ führt die Bildung regionaler Wirtschaftscluster im Wesentlichen auf das Bestreben der Unternehmen zurück, durch regionale Konzentration externe Effekte, u.a. einen intensivierten Informationsfluss hervorzurufen, der ihnen Vorteile im weltweiten Wettbewerb verschafft. Zur Entstehung dieser ballungsbedingten Wettbewerbsvorteile bedarf es einer „kritischen Masse" an Unternehmen eines wettbewerbsstarken Branchenschwerpunkts. Porter (1990) ging in seinen Überlegungen zunächst von der Bildung nationaler Wirtschaftscluster aus. Erst im Nachhinein nahm er die regionale Ebene in seine Betrachtung auf, wobei es weiterhin eher größere Gebiete wie z. B. die US-Bundesstaaten sind, in denen seiner Ansicht nach die erforderliche "kritische Masse" entstehen kann. Große Stadtregionen sind die weitaus kleinste Raumeinheit, in der er die Bildung eines regionalen Clusters für möglich hält (Porter 1998, S. 78).

$\mathrm{Zu}$ den Vorteilen der regionalen Agglomeration gehört insbesondere die Möglichkeit, persönliche Kontakte zu knüpfen und zu pflegen, z.B. zu Forschungseinrichtungen, Behörden und anderen Unternehmen. Die Wettbewerbsfähigkeit einer Region wird ebenso durch die Leistungsfähigkeit der dort ansässigen Menschen und Unternehmen geprägt, wie sie wiederum mit über den Erfolg der einzelnen Unternehmen entscheidet. Merkmale der regionalen Wettbewerbsfähigkeit sind z.B. die Qualifikation des Arbeitskräftereservoirs bzw. die Leistungsfähigkeit der Bildungseinrichtungen und die Intensität der Kooperation in wichtigen Branchen- schwerpunkten. Diese Eigenschaften spielen eine wachsende Rolle, denn gleichzeitig werden die Regionen in Bezug auf ihre Standorteigenschaften zunehmend "gläsern". Die Leistungsfähigkeit der Informations- und Kommunikationstechnologie ermöglicht es Unternehmen, Gründern und Arbeitnehmern, ohne großen Aufwand die verschiedenen Agglomerationsräume nach den für sie wichtigen Merkmalen miteinander zu vergleichen. Räume mit guten Lagevorteilen werden in der Tendenz durch Zuwanderung dieser Akteure "belohnt". ${ }^{3}$

Diese in Regionalforschung und Regionalpolitik anerkannte Argumentation führt zu der Schlussfolgerung, dass das Ruhrgebiet insgesamt oder sogar die "Metropolregion Rhein-Ruhr" den für das wirtschaftliche Handeln relevanten regionalen Bezugsrahmen darstellt, keinesfalls jedoch die einzelnen Städte, Landkreise und Gemeinden. Die Ausführungen im Folgenden beziehen zunächst Stellung zu der Frage, ob das Ruhrgebiet - oder in visionärer Diktion die „Metropole Ruhr“ einer stärkeren administrativen "Verklammerung" bedarf. Die weiteren Abschnitte befassen sich mit der Rolle des RVR sowie den Gestaltungsmöglichkeiten der Regionalplanung.

\section{Das RVR-Verbandsgebiet als „Metropole Ruhr"?}

Das Ruhrgebiet hat wie gesagt das Zeug, in Zukunft eine "Metropole Ruhr“ zu werden. Dieser Begriff ist sicher nicht einfach als bloßes Synonym für die ältere, an Kohle und Stahl erinnernde Gebietsbezeichnung „Ruhrgebiet“ zu verstehen. Stattdessen weckt „Metropole Ruhr“" Assoziationen an einen großstädtischen Wirtschaftsraum, der von hochinnovativen Industrien und Dienstleistungssektoren getragen und sowohl im Innern als auch von außen wesentlich als Einheit wahrgenommen wird (vgl. hierzu das engagierte Plädoyer für die „Weltstadt Ruhr von Christoph Zöpel 2005), bzw. gar an eine Megastadt mit einheitlicher Verwaltung, wie etwa London oder Paris. Die "Metropole Ruhr" ist im Reich des politischen Handelns jedoch bislang eher Wunsch und Vision denn gelebte Realität. Der Ballungsraum zwischen Rhein, Ruhr und Lippe wird sich nur dann zu einer Metropole entwickeln, wenn dieser Prozess einem einheitlichen, demokratisch-politischen Gestaltungswillen unterworfen wird. Dafür bedarf es einer administrativen Klammer um das Ruhrgebiet, so dass sich die Metropole Ruhr auch administrativ nach innen wie außen als Einheit darstellt. ${ }^{4}$ Dies ist weit mehr als eine Metropolregion Rhein-Ruhr im Sinne des Initiativkreises „Europäische Metropolregionen in Deutschland“" (vgl. hierzu Adam 2006; Steinacher 2006; 
Technische Universität Berlin 2003). Diesem Kreis geht es vor allem um die kommunenübergreifende Abstimmung von Raumplanungskonzepten und die Abstimmung der Investitionen in die Infrastrukturen. An eine administrative Integration ist jedoch kaum gedacht. Das Ruhrgebiet fungiert im Rahmen dieser Initiative zusammen mit den rheinischen Zentren Köln und Düsseldorf als "Metropolregion Rhein-Ruhr" (Blotevogel 2006).

Aktuell haben die auf eine stärkere Integration gerichteten Kräfte im Ruhrgebiet an Gewicht verloren und die auseinanderstrebenden, auf eine Lockerung des Zusammenhalts hinwirkenden Kräfte merklich an Gewicht gewonnen. Einzelne Kommunen der Ruhr-Agglomeration, insbesondere die bei der Bewältigung der Herausforderungen des wirtschaftlichen Strukturwandels eher erfolgreichen und an den Rändern der Agglomeration gelegenen, suchen stärker als in der Vergangenheit eigene Wege, verbunden mit der Option, sich früher oder später ganz aus dem Ruhrgebietsverbund herauszulösen. Die Bereitschaft, sich für einen großen städtischen Verbund zu engagieren, hat wohl im Ganzen merklich nachgelassen.

Für die stärkere Artikulation auseinanderstrebender Kräfte gibt es natürlich objektive Gründe. Die wirtschaftlichen Verflechtungen des polyzentrischen Ruhrgebiets wurden über ein Jahrhundert lang wesentlich von der Montanindustrie getragen. Die heutigen Großkommunen sind weitgehend um die Brennpunkte der Schwerindustrie herum gewachsen. Die Zusammenfas-

\section{Abbildung 2}

Sozialversicherungspflichtig Beschäftigte im Ruhrgebiet und im restlichen Nordrhein-Westfalen nach Sektoren - 1987 bis 2003 (Anteil in \%)

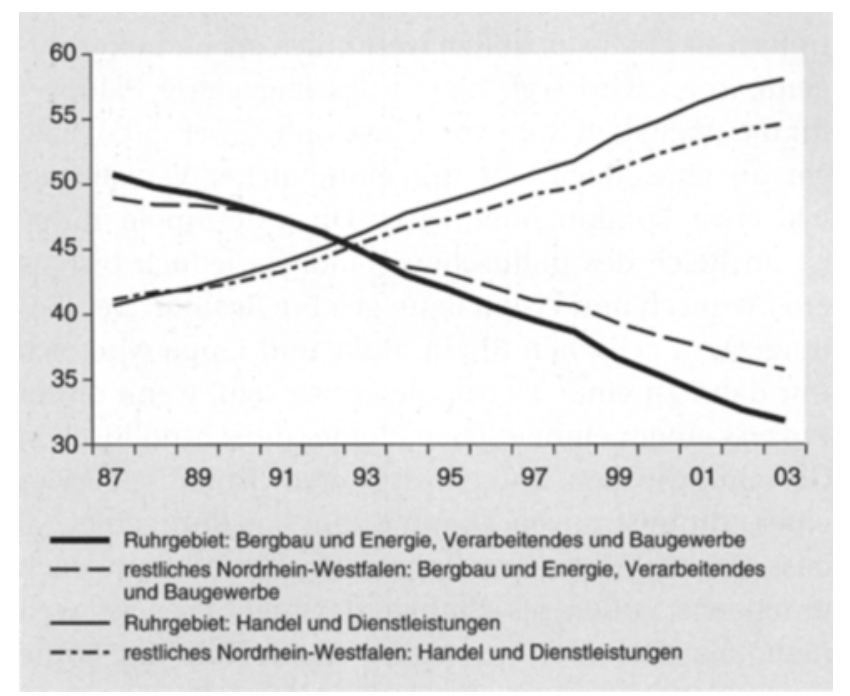

Quelle: eigene Berechnungen nach Angaben der Regionaldirektion der Arbeitsagentur NRW sungen der Produktionsstätten der Montanindustrie in Konzernen orientierten sich weitgehend an regionalen Gegebenheiten (vgl. Spethmann 1933, S. 528). Die kommunalen Reformen des 20. Jahrhunderts, aus denen die heutigen Großkommunen erwachsen sind, haben dann ihrerseits in starkem Maße die raumprägenden wirtschaftlichen Entwicklungen d s 19. Jahrhunderts auf administrativer Ebene nachvi lzogen. Kohle und Stahl nebst zuliefernden Industrien stellten das „Bindemittel“ dar, das das Ruhrgebiet wirtschaftlich zusammenhielt und die Arbeitsmarktverflechtungen zwischen den kreisfreien Städten des Reviers bestimmte. Diese räumlichen Konstellationen der Arbeitsmärkte in der Region haben jedoch an Bindungskraft verloren (vgl. Abb. 2).

Eine nicht mehr ganz aktuelle Analyse der Entwicklung der regionalen Arbeitsmärkte im Zeitraum 1987 bis 1998 wies bereits auf erhebliche Veränderungen der Arbeitsmarktverflechtungen im Ruhrgebiet und in seinen „Außenkontakten“ mit den Nachbarregionen hin. Die Pendlerströme über die RVR-Grenzen hinweg haben - aus dem Ruhrgebiet heraus und in dieses hinein - zugenommen, insbesondere die mit der Landeshauptstadt Düsseldorf. ${ }^{5}$ Zugleich haben sich die Pendlerströme innerhalb des Ruhrgebiets im Ganzen intensiviert. Eine neue Untersuchung zeigt, dass sich diese Entwicklungen bis 2002 weiter fortsetzten (Neumann/Tonks 2007).

So ist zwar z.B. Düsseldorf der wichtigste Zielort der Berufs- und Ausbildungspendler aus Duisburg. Jedoch überwiegt insgesamt der Auspendlerstrom aus Duisburg in die anderen Gemeinden des RVR-Gebiets mit (2002) 44949 Auspendlern deutlich gegenüber dem nach Düsseldorf (17 366 Auspendler). Im Zeitraum von 1998 bis 2002 nahmen die Pendlerströme aus Duisburg sowohl in Richtung Rheinland als auch ins östliche Ruhrgebiet, etwa nach Dortmund und Herne deutlich zu (Abb. 3).

Die ehemals gegebene ausgeprägte Fokussierung auf die Kernstädte hat nachgelassen. Hierüber schon das „Ende des Ruhrgebiets“ im Sinne der Auflösung jeglichen Zusammenhalts der Region zu verkünden, wäre freilich viel zu weit gegriffen und würde die partikularistischen Tendenzen, die es ja in diesem Agglomerationsraum seit dem Siegeszug der-Industrialisierung im 19. Jahrhundert schon immer gab, deutlich überzeichnen. Jenes Ruhrgebiet, das die große industrielle Vergangenheit Nordrhein-Westfalens verkörperte, ganz von Kohle und Stahl lebte und in dem die Infrastrukturen und Verwaltungsfunktionen auf die Bedürfnisse der Montanindustrie zugeschnitten waren, existiert allerdings längst nicht mehr. Die Politik hat dies mit dem jetzt vereinbarten Ausstieg aus den Kohlesubventionen 


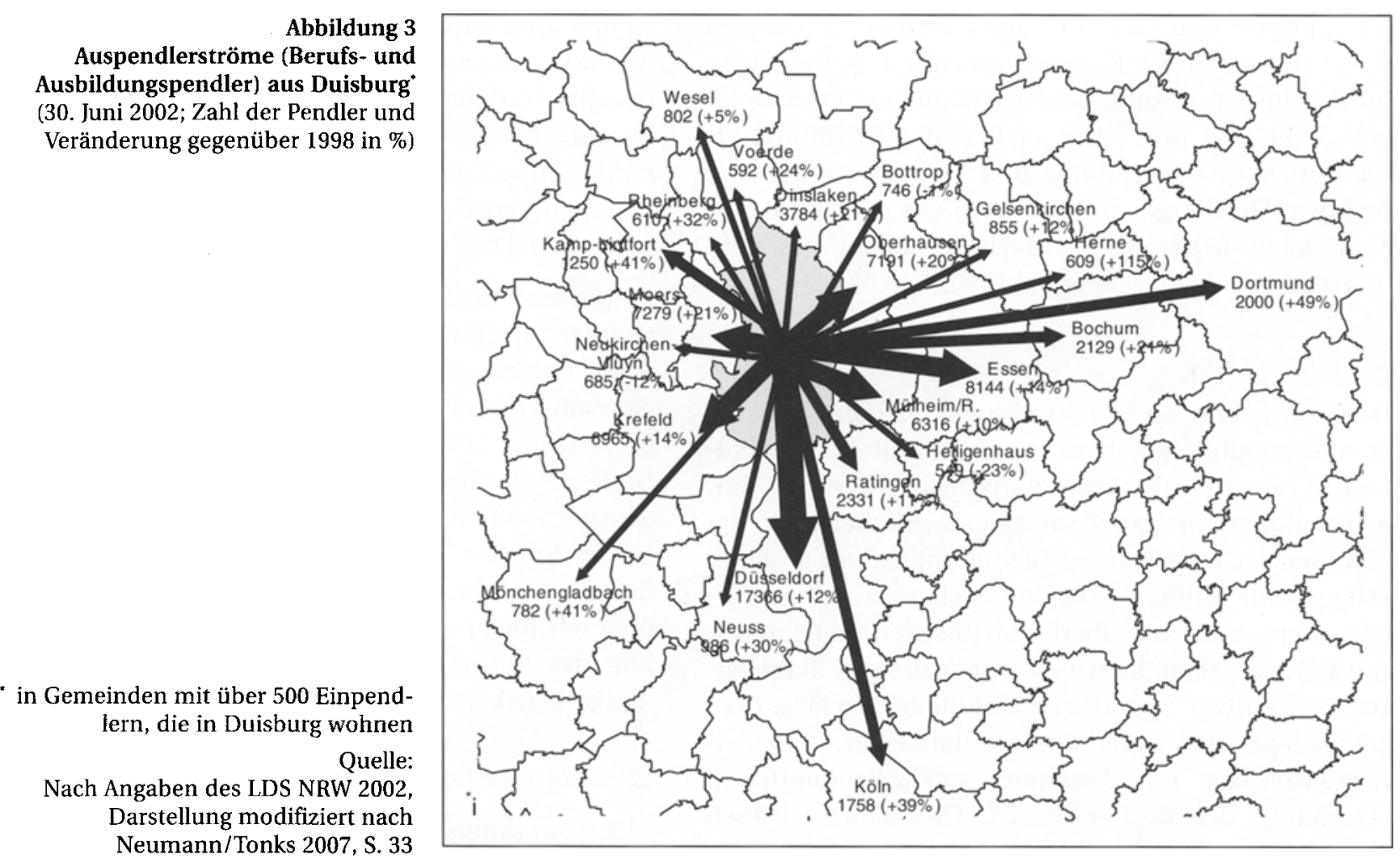

- wenn auch aus gesamtwirtschaftlicher Sicht viel zu spät - ihrerseits offiziell bestätigt.

Die große städtische Agglomeration „Ruhr“ existiert innerhalb des RVR-Gebiets auf neuen wirtschaftlichen Grundlagen aber sehr wohl und zeichnet sich trotz aller fortbestehenden gravierenden Strukturprobleme durch eine bemerkenswerte Vitalität aus. Es ist in diesem $\mathrm{Zu}$ sammenhang durchaus sinnvoll, die Gemeinsamkeiten der Agglomeration an Rhein und Ruhr zu betonen und - zukunftsorientiert sowie optimistisch - von der „Metropole Ruhr" zu sprechen. Drei Argumente sprechen trotz der Auflösung des Montankomplexes für den real bestehenden wirtschaftlichen und gesellschaftlichen Zusammenhalt des Ruhrgebiets:

(1) wirtschaftliche und infrastrukturelle Verflechtungen unabhängig von den beschriebenen Pendlerströmen

(2) das Gefühl der Zugehörigkeit zur Region und ihre Wahrnehmung von außen

(3) die kulturelle Prägung des Ruhrgebiets als einheitlicher, sich deutlich vom übrigen Westfalen und Rhein land abhebender Raum.

Darüber hinaus ist der administrative Zusammenhalt des Ruhrgebiets auch aus strategischen Gründen wünschenswert: Ein großer städtischer Ballungsraum verfügt über Chancen im europäischen und globalen Standortwettbewerb, jede einzelne Ruhrgebietsstadt für sich genommen jedoch nicht.

\section{(1) Verflechtungen}

Es wäre ein Fehler, den intraregionalen wirtschaftlichen Zusammenhang des Ruhrgebiets ausschließlich an den - wie erläutert intensiven - Arbeitsmarktverflechtungen, d.h. den Arbeitspendlerströmen zwischen den Kommunen zu messen. Zwischen den Unternehmen, insbesondere den Großunternehmen der Region bestehen vielfältige Kapitalverflechtungen. Diese sind nach Wissen der Verfasser bislang noch nicht systematisch untersucht worden. Manches spricht aber dafür, dass sie innerhalb des Ruhrgebiets stärker ausgeprägt sind als mit anderen städtischen Zentren des Landes. Auch die Unternehmen neuer innovativer Industrien (einschl. Dienstleistungsbranchen) kümmern sich bei ihrer Standortwahl wenig um Gemeinde- und Kreisgrenzen. Hier sind zumindest stellenweise Strukturbildungen über Kommunen hinweg zu beobachten, beispielsweise in der Mikrosystemtechnik, im Logistiksektor und sicher auch in der Gesundheitswirtschaft. Schließlich ignorieren die Konsumenten in ihrem Einkaufsverhalten die kommunalen Grenzen. In den steigenden Pendlerströmen der Verbraucher spiegeln sich die zunehmenden Präferenzen für überregionale Einkaufszentren wider. Neben dem Warenangebot kommt der bereitgestellten Infrastruktur besondere Bedeutung zu. Prominentestes neueres Beispiel für erfolgreiche überregionale Einkaufszentren ist das CentrO Oberhausen, das sich eines regen Zuspruchs aus der wei- 
teren Region und nicht nur aus Oberhausen und den direkt benachbarten Kommunen erfreut. Befragungen im Duisburger Norden haben z. B. aufgezeigt, dass im Jahr 1999, also drei Jahre nach seiner Eröffnung, für die dort ansässige Wohnbevölkerung das CentrO neben dem Duisburger Stadtzentrum das wichtigste Einkaufsziel für Güter des nichttäglichen Bedarfs darstellte (Neumann/Schatz-Bergfeld 1999).

\section{(2) Zugehörigkeit}

Das Ruhrgebiet wird in Nordrhein-Westfalen und im gesamten Bundesgebiet trotz der tiefgreifenden Wandlungen seiner Wirtschaftsstruktur nach wie vor als Einheit wahrgenommen. Zwar spielen hierbei trotz der IBA und der respektablen Bemühungen, ein anderes, zeitgemäßes Antlitz der Region nach außen zu vermitteln, noch allzu oft überholte Klischeevorstellungen von Kohlebergbau, konventionellen riesigen Stahlwerken und Luftverschmutzung eine Rolle, die längst ein Zerrbild der realen Verhältnisse darstellen - ein Zeichen, dass es eines offensiveren, zukunftsorientierten Marketings der Region bedarf. Dies ändert jedoch nichts an der Tatsache, dass das Ruhrgebiet als Ganzes eine feste Größe in der öffentlichen Wahrnehmung bildet. Die kleineren kreisfreien Städte und die kreisangehörigen Kommunen des RVR-Verbunds haben dem nichts entgegenzusetzen. Die größten Kommunen - Dortmund, Essen, Duisburg, Bochum - haben zwar eine reelle Chance, landes- und bundesweit als eigenständige Einheiten wahrgenommen zu werden, mit einer „Metropole Ruhr“ können sie aber allemal nicht konkurrieren.

Zugleich ist davon auszugehen, dass in der Bevölkerung des Ruhrgebiets ein Gefühl der Zugehörigkeit zu einem städtischen Ballungsraum mit großer Industriegeschichte, der in Zeiten eines schwierigen Strukturwandels seinen Weg in die wissensbasierte Ökonomie des 21. Jahrhunderts sucht, weit verbreitet ist. Wie Blotevogel (2001, S. 16-17) feststellt, ist zwar unter regionalen Eliten noch eine gewisse Distanzierung gegenüber dem vermeintlich proletarischen und provinziellen Ruhrgebiet festzustellen, hat sich diese Einstellung jedoch in den jüngeren Generationen deutlich hin zu einer stärkeren Identifikation mit dem Ruhrgebiet geändert. Eine wesentliche Rolle scheint seiner Ansicht nach dabei das „... dramatisch gewandelte Verhältnis zur industriell geprägten Kulturlandschaft zu spielen“. Vielleicht ist die folgende Aussage von Ebert et al. (2005, S. 10) noch etwas verfrüht: „Der ,Pott' ist heute Kult; die einst negativ konnotierte Bezeichnung der von Kohle und Stahl geprägten Industrieregion an Rhein und Ruhr trägt heute alle Merkmale einer Marke." Manches spricht aber dafür - z.B. die Verkehrsströme zwischen den Kommunen -, dass zumindest die nachwachsenden Generationen sich in ihrem durch ein hohes Maß an individueller Mobilität gekennzeichneten Arbeits-, Freizeit- und Konsumverhalten stark regional und eben nicht nur an der jeweiligen Großkommune oder an einem Ortskern innerhalb der Kommune orientieren.

Empirische Befunde darüber, wie die Ruhrgebietsbewohner ihr Umfeld (Ortsteil, Kommune, Ruhrgebiet) wahrnehmen und wie sie die Entwicklung in der Region einschätzen, sind allerdings rar. Eine im September/Oktober 2001 durchgeführte "große RuhrgebietsUmfrage" des Bochumer Instituts BIFAK dokumentiert, dass sich - wenig überraschend - die jeweilige Stadt als wichtigster identitätsstiftender Bezugspunkt der Befragten erwies. ${ }^{6}$ Diese zeigten sich jedoch gegenüber der Idee einer stärkeren administrativen Integration des Ruhrgebiets bemerkenswert aufgeschlossen (Stuke/Kruck 2002, S. 128-138, 146).

\section{(3) Kulturelle Prägung}

Nicht zu unterschätzen sind schließlich langfristige kulturelle Prägungen, die sich seit der Industrialisierung der Region im 19. Jahrhundert gebildet haben und in der Gegenwart in veränderter Form weiter wirksam sind. So wurde in der Ruhrgebietsforschung bisweilen eine durch die Erfahrungen der Montanindustrie geprägte Mentalität der Bevölkerung als Hemmnis für die Bewältigung des Strukturwandels ins Feld geführt, die sich z. B. in besonders niedrigen Selbständigenquoten äußere. Schließlich weist die Kulturszene des Ruhrgebiets eine bemerkenswerte Fülle von Einrichtungen überregionaler Bedeutung auf. In der Tat hat sich im Ruhrgebiet in den letzten Jahrzehnten eine Vielzahl von Wirtschaftsaktivitäten stärker etabliert, die als Querschnittsbranche "Kulturwirtschaft" zusammengefasst bereits ein beachtliches Gewicht aufweisen (vgl. Ebert et al. 2005). In seiner Gesamtheit kann das Ruhrgebiet durchaus mit den großen deutschen Kulturzentren wie München und Hamburg konkurrieren; die einzelnen Städte können dies in der Regel kaum.

Die bisherige Argumentation führt $\mathrm{zu}$ der grundsätzlichen Frage der Regionalforschung und -politik, wie Regionen vernünftigerweise abgegrenzt werden sollten. Ohne Zweifel sollte man solche Gebietseinheiten zusammenführen, die auch „zusammenpassen“ (Maier/Tödtling 2002). Zur Bestimmung der Zusammengehörigkeit kommen zwei Kriterien in Frage: Homogenität und Verflechtung. Nach dem ersten Kriterium werden Regionen gebildet, deren Teilgebiete sich nach bestimmten Indikatoren sehr ähnlich sind. Das 
zweite Kriterium fügt solche Gebiete zusammen, die - z.B. an Pendlerströmen oder am Einkaufsverhalten gemessen - in besonders enger Beziehung zueinander stehen. In den Regionalwissenschaften wird es auch als „Funktionalitätskriterium“ bezeichnet. Das Homogenitätskriterium spricht eindeutig für die „Metropole Ruhr“ als regionalpolitische Bezugseinheit und gegen eine Zusammenfassung zur Metropolregion RheinRuhr. Die funktionale Region geht sicher über das RVR-Gebiet hinaus. Ihr gehört einerseits ein weiterer Umlandbereich an, andererseits ist sie, wie erläutert, über die Pendlerströme eng mit der Landeshauptstadt verbunden. Selbstverständlich spricht neben vielen anderen Argumenten auch die intensive Nutzung des Düsseldorfer Flughafens durch Bevölkerung und Unternehmen des Ruhrgebiets für die engen Beziehungen zur Landeshauptstadt. Offensichtlich folgen die Städte und Gemeinden des Ruhrgebiets aber doch einem regionalen "Entwicklungspfad“ (Martin/Sunley 2006), der sich von dem der Landeshauptstadt Düsseldorf so sehr unterscheidet, dass in der näheren Zukunft die vergleichsweise homogenere Region Ruhr einen sinnvolleren regionalpolitischen Bezugsrahmen bildet.

Von der Existenz einer Vielzahl von wirtschaftlichen, infrastrukturellen, sozialen und kulturellen Bindungen der städtischen Agglomeration Ruhr, die eine geeignete Grundlage für eine stärkere administrative Integration des Ruhrgebiets bilden könnten, sofern der politische Wille hierfür bei den Akteuren auf kommunaler und Landesebene vorhanden ist, besteht somit kein Zweifel. Das wichtigste Argument für ein stärkeres Zusammengehen der Kommunen und Kreise bezieht sich jedoch weniger auf in der Vergangenheit geprägte Gemeinsamkeiten als vielmehr auf die realen Chancen, die eine stärkere Integration im nationalen, europäischen und globalen Standortwettbewerb mit sich bringt: Eine „Metropole Ruhr“ hat ungleich bessere Möglichkeiten, sich im internationalen Standortwettbewerb zu behaupten als jede einzelne Kommune für sich genommen. Eine "Weltstadt Ruhr" wird auch in Kalifornien, Mumbai oder Shanghai als solche wahrgenommen, Dortmund, Essen oder Duisburg - um nur die größten Ruhrgebietsstädte zu nennen - dagegen wohl kaum. In Zeiten, in denen überall in den „Metropolregionen“ Europas - so z. B. in der niederländischen Randstad ${ }^{7}-$ über Integrationsdefizite und eine engere administrative Verzahnung nachgedacht wird ${ }^{8}$, sollte dies auch für die politisch Verantwortlichen im Ruhrgebiet und im Land kein unüberwindbares Problem sein.

\section{Bessere administrative Integration: die Rolle des RVR}

Die Überleitung des Kommunalverbands Ruhrgebiet (KVR) in den Regionalverband Ruhrgebiet (RVR) am 1. Oktober 2004 stellt das momentane (Zwischen-)Ergebnis einer politisch kontrovers geführten Diskussion über die Schaffung adäquater Verwaltungsstrukturen für den größten deutschen Ballungsraum dar. Angeregt durch landespolitische Diskussionen über eine Reform der Verwaltungszuständigkeiten für das Ruhrgebiet, kam es Ende der 1990er Jahre zu einer vom KVR unterstützten Argumentation für eine „Ruhrstadt“, d.h. eine Fusion der Städte und Gemeinden des Ruhrgebiets (Willamowski 2001). Bis heute handelt es sich dabei mehr um eine inner- wie außerhalb des Ruhrgebiets umstrittende Vision als um eine konkrete Perspektive für die nähere Zukunft. Im Jahr 2000 gründete die damalige Landesregierung die - mit weitreichenden strukturpolitischen Kompetenzen ausgestattete - Projekt Ruhr GmbH. Angesichts einer offensichtlich nicht an der Stärkung des KVR interessierten Landesregierung akzeptierten die Verantwortlichen des KVR, wie Voß $(2007$, S. 8) feststellt, ,aus Angst, ganz aufgelöst zu werden, ... eine Kompetenzreduktion, die dem Vorwurf seiner faktischen Wirkungslosigkeit immer noch mehr Nahrung geben musste". Zur Auflösung des KVR ist es nicht gekommen. Die 2007 verabschiedeten Änderungen des Landesplanungsgesetzes NRW (s.o.) und des RVR-Gesetzes (s.u.) haben die Rolle des RVR gestärkt. Die Projekt Ruhr GmbH wurde zum 31. Dezember 2006 aufgelöst, Teile ihrer Aufgaben übernahm der RVR.

Die Übertragung der Zuständigkeit für die Regionalplanung auf den RVR ist aus organisatorischer Sicht logisch gerechtfertigt. Der RVR verfügt aufgrund seiner eigenen Vergangenheit und der seiner Vorläufer Kommunalverband Ruhrgebiet (KVR) und Siedlungsverband Ruhrkohlebezirk (SVR) über einschlägige Erfahrungen in Planung wie Koordination (zum RVR vgl. z. B. Kühn 1996). Er erstellt schon bisher per gesetzlichem Auftrag „Planungs- und Entwicklungskonzepte für das Verbandsgebiet (Masterpläne), die als Ziele der Regionalentwicklung des Verbandsgebiets bei der Aufstellung der Bauleitpläne der Mitglieder des Verbandes" und bei der Erarbeitung der Gebietsentwicklungspläne sowie von regionalen Flächennutzungsplänen zu berücksichtigen sind ( $\$ 6$ RVR-Gesetz). Zugleich gibt es kein anderes kommunenübergreifendes Gremium, das diese Aufgabe ohne Weiteres übernehmen könnte. Die Einrichtung eines mit Planungsbefugnissen ausgestatteten Regierungsbezirks Ruhr, der über eine eigene Bezirksplanungsbehörde verfügen würde, ist derzeit 
nicht in Sicht, die Schaffung einer gänzlich neuen interkommunalen Planungseinrichtung wäre angesichts des Vorhandenseins einer solchen funktionstüchtigen Einrichtung nicht sinnvoll.

Freilich wird dem RVR mit der geplanten gesetzlichen Neuregelung „nur“ eine Kompetenz zurückgegeben, die sein Vor-Vorgänger SVR im 20. Jahrhundert schon einmal über Jahrzehnte hinweg besessen hatte. Bereits die Gründung des SVR im Jahr 1920 hatte zum Ziel, die ortsübergreifenden Aufgaben des Ruhrgebiets in regionaler Selbstverwaltung zu bündeln (Pankoke 1990, S. 16-17). Auf einen festgestellten regionalen Steuerungsbedarf hatten zuvor schon die ersten Zweckverbände wie die Emschergenossenschaft und der Verband zur Reinhaltung der Ruhr reagiert. Was in der Weimarer Republik und nach der Katastrophe des „Dritten Reichs“ im sich neu konstituierenden SVR in der Bundesrepublik leidlich gut funktionierte, wurde durch die Gebietsreform der Jahreswende 1974/75 zunichte gemacht. Die bislang dem SVR übertragenen Kompetenzen der Landesplanung wurden in den staatlichen Verfügungsbereich reintegriert (Pankoke 1990, S. 64).

Diese Entscheidung war zwar vor dem Hintergrund der Gebietsreformen der Nachkriegsjahrzehnte, die zur Bildung immer größerer und damit auch für die Bürger „entfernterer“ kommunaler Einheiten führten, verständlich. Die Großkommunen des Ruhrgebiets waren auf Handlungsfreiheit erpicht und wenig geneigt, ein Stück ihrer Autonomie an eine „übergeordnete" Einheit - den SVR, später den KVR - abzugeben. Für das Ruhrgebiet als Ganzes freilich bedeutete der Verlust der Regionalplanungskompetenz, dass die Chance einer selbst verantworteten Steuerung der Raumentwicklung der Region gerade in der kritischen Zeit des Strukturwandels der Montanindustrie verspielt war. Die Aufgabe der Wahrnehmung der ganzheitlichen Ruhrgebietsinteressen fiel unter der neuen Verwaltungskonstruktion der Landesregierung zu. In der Rückschau handelt es sich wohl um eine gravierende Fehlentscheidung.

Die Übertragung der Regionalplanungskompetenz auf den RVR kann ihren Zweck allerdings erst dann voll erfüllen, wenn dieser auch mit entsprechenden finanziellen und personellen Ressourcen ausgestattet wird sowie die Entscheidungshoheit über die zur Umsetzung der Regionalplanung nötigen Finanzmittel erhält. Angesichts des Gebots eines sparsamen Umgangs mit Steuermitteln müsste dabei die Ressourcenausstattung untergeordneter Verwaltungsebenen Zug um Zug zurückgefahren werden. Hier sehen die Autoren einen Schwachpunkt des Gesetzentwurfs. Die vernünftige und zukunftsweisende Vision einer „Metropole Ruhr" steht somit in einem gewissen Kontrast zu dem eher bescheidenen Charakter des mit dem Gesetzentwurf anvisierten Ziels der Übertragung der Regionalplanungskompetenz an den RVR. Damit wird zwar ein neues, jedoch kein revolutionäres Kapitel in den mittlerweile über 100 Jahre zurückreichenden $\mathrm{Be}$ mühungen aufgeschlagen, dem Ruhrgebiet einen engeren administrativen Zusammenhalt zu verleihen.

Die Regionalplanung für die "Metropole Ruhr“ sollte sich vor allem mit solchen Fragen befassen, die für die Region als Ganzes von Interesse sind und deren befriedigende Lösung ihre Attraktivität in den Augen einheimischer und externer Investoren erhöhen können. Dies betrifft z.B. die Verbesserung der Verkehrsinfrastruktur in der polyzentrischen Agglomeration Ruhrgebiet, die Weiterentwicklung der in den vergangenen Jahrzehnten auf den Weg gebrachten, bereits recht erfolgreichen ökologischen Sanierungsprojekte und die Abstimmung von Entwicklungsprojekten mit überkommunaler Bedeutung. Regionalplanung kann allerdings nur dann erfolgreich sein, wenn sie die kommunale Planung ergänzt und von den beteiligten kreisfreien Städten und Kreisen akzeptiert wird. Die Regionalplanungsinstanz RVR steht hier vor der nicht allein durch rechtliche Vorkehrungen abzusichernden, sondern auch von allseitiger Akzeptanz und politischem Geschick abhängigen Gratwanderung zwischen kommunalem Eigeninteresse und den Interessen der "Metropole Ruhr“ als Ganzes. In jüngerer Vergangenheit konnten innerhalb des Ruhrgebiets Erfahrungen in der interkommunalen, projektbezogenen Zusammenarbeit u.a. im Rahmen der IBA Emscher Park gewonnen werden. Wie Kilper (1995) darlegt, stellten die IBA-Projekte die Kommunen vor ungewohnte organisatorische Herausforderungen. Die im Zuge der IBA erzielten städtebaulichen und umweltbezogenen Verbesserungen haben wesentlich zur Veränderung der Wahrnehmung des Ruhrgebiets beigetragen, innerhalb wie außerhalb der Region. Das Beispiel IBA belegt, dass die projektorientierte Verwirklichung von Ruhrgebietsinteressen unter aktiver Mitwirkung der Kommunen möglich ist.

Die Zukunftsfähigkeit der „Metropole Ruhr“ hängt vor allem von der Herausbildung überregional und international wettbewerbsfähiger, innovationsstarker Branchenschwerpunkte ab. Diese Branchenkerne entstehen z.B. im Umfeld wichtiger Forschungseinrichtungen und beschränken sich in ihrer Standortwahl innerhalb des Ruhrgebiets keineswegs auf das Gebiet einer einzelnen Kommune. Es kommt somit der Denkweise ökonomischer Entscheidungsträger entgegen, wenn man sich bei Anstrengungen zur Förderung der örtlichen Standortbedingungen strategisch nicht nur auf die einzelnen Städte konzentriert. Auch hochqua- 
lifizierte Arbeitnehmer ziehen bei ihrer Entscheidung über einen möglichen Zuzug die Attraktivität der Region insgesamt in Betracht.

Hierbei stellt die dezentrale, polyzentrische Struktur des Ruhrgebiets im Vergleich zu den auf ein Zentrum hin orientierten Agglomerationen eine Besonderheit dar. Diese wirkt sich unter den Bedingungen der heute gegebenen administrativen Fragmentierung eher als Nachteil für die Region als Ganzes aus. Standortentwicklungsprojekte, die die Kraft der einzelnen Kommunen überschreiten, können nicht realisiert und die potenzielle Attraktivität der gesamten Region für auswärtige Investoren kann nicht voll zur Geltung gebracht werden. An diesem Schwachpunkt setzt die Übertragung der Regionalplanungskompetenz auf den RVR an. In die gleiche Richtung zielen die Bemühungen des RVR um ein regionales Standortmarketing sowie die Schaffung einer überregionalen Wirtschaftsförderungseinrichtung. Was sich zunächst als Nachteil für das Ruhrgebiet darstellt - seine polyzentrische Struktur -, könnte sich so im günstigen Fall als Stärke erweisen. Es wäre ideal, wenn man einen vernünftigen Kompromiss zwischen der Entfaltung der Stärken des Ruhrgebiets als Ganzes und einem lokalen Standortwettbewerb der Ruhrgebietsstädte finden könnte, der der Durchsetzung gemeinsamer Belange nicht im Wege steht - also das Zusammenspiel von Wettbewerb und Kooperation unter Berücksichtigung des Subsidiaritätsprinzips.

Die Übertragung der Regionalplanungskompetenz für die „Metropole Ruhr" auf den RVR ist somit als wichtiger Schritt zur stärkeren Berücksichtigung gemeinschaftlicher, kommunenübergreifender Belange im Ruhrgebiet zu begrüßen.

\section{Bedeutung der Regionalplanung für die „Metropole Ruhr“}

Die Übertragung der Regionalplanungskompetenz auf den RVR ist vor diesem Hintergrund eine ebenso konsequente wie richtige Entscheidung für die Schaffung vorteilhafter Standortbedingungen in der Gesamtregion. Der Regionalverband Ruhrgebiet verfügt über ein eigenes Regionalparlament, das proportional $\mathrm{zu}$ den Ergebnissen der Kommunalwahl besetzt wird. So ist es nur konsequent, diesem Organ die Zuständigkeit für die räumliche Entwicklung seines Verwaltungsgebiets zu übertragen und nicht, wie es im Ruhrgebiet bis zum Inkraftreten der Gesetzesänderung des Landesplanungsgesetztes im Jahr 2009 noch der Fall sein wird, auf die zuständigen Regierungspräsidien zu verteilen. Ohne Zweifel ist das Ruhrgebiet vor allem durch seine wirtschaftlichen Standortfaktoren als eine regio- nale Einheit zu betrachten, deren Förderung Aufgabe einer für dieses Gebiet zuständigen Regionalplanung sein sollte. ${ }^{9}$

Für die bis auf die Planungshoheit im Ruhrgebiet unangetastet bleibenden Regierungsbezirke ergibt sich durch den regionalen Neuzuschnitt ihrer Planungskompetenz ebenfalls die Chance, sich bei der Gestaltung der Rahmenbedingungen des wirtschaftlichen Handelns zielgerichteter auf ihre siedlungsstrukturell nun kohärenteren Gebiete zu fokussieren. Allerdings sind die Einschätzungen zur staatlichen Gestaltungsmöglichkeit des Wirtschaftsraums grundsätzlich nicht eindeutig. Die modernen Wirtschaftswissenschaften stehen dem etwas skeptisch gegenüber, Teile der "Scientific Community“ der Raumplaner hingegen scheinbar überaus optimistisch. Manche Dissonanz geht hierbei wohl eher auf bloße Missverständnisse zwischen zwei sich aus unterschiedlichen Denktraditionen speisenden akademischen Kulturen zurück (ähnlich Peck 2003).

Aus Sicht der Autoren ist die Regionalplanung für das Ruhrgebiet somit nicht als Versuch zu verstehen, die wirtschaftliche Entfaltung der Region im Sinne von dirigistischen Planungsvorstellungen $\mathrm{zu}$ „steuern“. Vielmehr geht es darum, in einem dicht besiedelten Agglomerationsraum für gute, homogene Infrastrukturen zu sorgen und ein gutes Zusammenspiel verschiedener Flächennutzungen zu erreichen, z.B. Industrie, Einkaufen, Wohnen, Freizeit, Kultur, Naherholung. Moderne Konzepte der Raumordnung gehen ebenfalls davon aus, dass es Aufgabe der Raumentwicklungspolitik ist, regionalwirtschaftliche Spezialisierungen zu unterstützen, ohne sie „lenken“ oder gar „erzeugen“ zu können (BMVBS 2006). Dem „aktivierenden Staat“ kommt in diesen Konzepten vor allem die Aufgabe zu, Impulse für eine netzwerkartige regionale Selbstorganisation zu vermitteln (Regional Governance, Fürst 2001).

Gegen eine solche Beeinflussung der wirtschaftlichen Rahmenbedingungen ist aus regionalökonomischer Sicht nichts einzuwenden. So findet auch in wirtschaftsliberalen Staaten wie den USA oder Großbritannien eine vielgestaltige staatliche Einflussnahme auf die Flächennutzung statt, die keineswegs verhaltener ist als in den korporatistisch geprägten kontinentaleuropäischen Wirtschaften. Es gilt, Überlegungen zur Regionalentwicklung durch Bestimmung konkreter Raumnutzungen „in den Boden zu übersetzen“. Das „Verbandsmodell“ der Regionalplanung erscheint dabei als eine für die Rahmenbedingungen des polyzentrischen Agglomerationsraums Ruhrgebiet akzeptable, weil auf Selbstverwaltungsbasis durch die Kommunen beruhende Kompromisslösung zwischen kommunaler 
Fragmentierung und weitergehender Integration. Das Ruhrgebiet verfügt zwar nicht mehr über eine einheitliche montanindustriell geprägte Wirtschaftsstruktur. Der siedlungsräumliche Bestand geht jedoch auf die in der Vergangenheit einheitliche Wirtschaftsentwicklung zurück, aufgrund derer es zur Bildung von wirtschaftsräumlichen Zonen kam. So ist z.B. die Siedlungs- und Bevölkerungsstruktur des Duisburger Nordens eher mit der des Essener Nordens zu vergleichen als mit der des Duisburger Südens.

Zur Schaffung vorteilhafter wirtschaftlicher Standortbedingungen ist wie erläutert aus regionalökonomischer Sicht die Bildung einer regionalen „Klammer“ zu begrüßen. $\mathrm{Ob}$ es im Laufe des 21. Jahrhunderts zu vertieften Formen einer verwaltungsmäßigen Verzahnung der Städte und Kreise des Ruhrgebiets kommen wird, bleibt allerdings abzuwarten. Die raumwirtschaftliche Logik und die Herausforderungen der wissensbasierten Ökonomie in einer globalisierten Weltwirtschaft sprechen zwar eindeutig dafür. Die Erfahrungen des 20. Jahrhunderts mit regionaler und kommunaler Ruhrgebietspolitik stimmen jedoch recht skeptisch, dass dies in absehbarer Zeit gelingen könnte.

Butzin/Franz/Noll (2006) sehen im Management der Flächen(nutzung) ein Schlüsselelement zur Flankierung der kommenden demographischen und wirtschaftlichen Veränderungen, die das Ruhrgebiet betreffen. Sie regen einerseits die Entwicklung neuer Formen der $\mathrm{Pu}$ blic-Private-Partnership an, z. B. öffentlich finanzierte Zwischennutzungen auf momentan nicht vermarktungsfähigen Brachflächen privater Grundeigentümer, um das Auftreten innerstädtischer „Siedlungslücken“ zu vermeiden. Zum anderen fordern sie eine Einbettung der Flächenbausteine in langfristige, auf die interregionale und globale Maßstabsebene gerichtete Strategien. Sie sehen es als Chance der Regionalplanung an, die räumliche Clusterbildung im Ruhrgebiet durch ein auf die Förderung transurbaner Vernetzungen zielendes Flächenmanagement zu unterstützen, und verweisen auf eine entsprechende Planungspolitik skandinavischer Erfolgsregionen. Der von Florida (2005, S. 61) untersuchte Zusammenhang zwischen Umweltqualität und Standortwahl der Hightech-Industrien und ihrer Beschäftigten, der sog. „creative class“, wird von ihnen als Argument dafür herangezogen, die Entwicklung attraktiver innerstädtischer Wohnflächen eng mit der der Erschließung von Gewerbeflächen für Wirtschaftscluster zu verknüpfen. Als Beispiel nennen sie das "dortmund-project", das neben der Umnutzung ehemaliger Stahlwerkflächen zu Hightech-Standorten umfassende städtebauliche Aufwertungsmaßnahmen vorsieht, z.B. die Schaffung einer neuen „Waterfront“ in Form des Phoenixsees.
Aus Sicht der Verfasser ist gerade der zweite von Butzin/Franz/Noll genannte Strategiebereich ein Kernargument für die Stärkung der regionalen Planungsebene in der „Metropole Ruhr“. Der Wissensstand über die Voraussetzungen zur Schaffung wettbewerbsfähiger Wirtschaftscluster (s.o.) legt nahe, dass eine "kritische Masse“ an innovativen Unternehmen und Existenzgründern in Zukunftsbranchen kaum in einer einzelnen Stadt des Ruhrgebiets auftreten wird. Der Regionalplanung sollte nach aktuellen Vorstellungen über die Rolle der Raumentwicklungspolitik vor allem die Aufgabe zukommen, projektorientierte Förderprogramme, die über einen befristeten Zeithorizont hinweg auf die Aktivierung vorhandener Stärken abzielen, durch ein entsprechend flexibles Flächenmanagement zu unterstützen. Dieses Verständnis von Regionalplanung liegt auch den „Leitprojekten“ zugrunde, die im Rahmen des Forschungsvorhabens "Städteregion Ruhr 2030 “ von 2001 bis 2003 von den Städten Bochum, Dortmund, Duisburg, Essen, Gelsenkirchen, Herne, Mülheim/Ruhr und Oberhausen in Kooperation mit der Fakultät Raumplanung der Universität Dortmund für das Gebiet dieser Städte konzipiert wurden. Als Leitprojekte werden u.a. die stadtregionale Planung (themenbezogene Masterpläne und regionale Flächennutzungspläne) sowie die kooperative Entwicklung und Vermarktung gewerblicher Flächen identifiziert, außerdem die Aufwertung der Uferlagen ( $z$. B. als "Avantgarde des neuen Wohnens"), die stadtregionale Migrationspolitik und die Haushaltskonsolidierung (Davy 2004, S. 19).

Eine zukunftsorientierte regionalplanerische Einwirkung auf die Entwicklung der Metropole Ruhr sollte folgenden Leitlinien folgen:

(1) Es wird nicht möglich sein, durch regionalplanerische Einflussnahme die Entwicklung eines Wirtschaftsraums zu „steuern“. Seine Gestaltung hängt von den Entscheidungen individueller ökonomischer Akteure ab, d.h. von Unternehmen und Einzelpersonen. Aus Sicht der Wirtschaftswissenschaften verhält sich der einzelne Mensch angesichts allumfassender Knappheiten als rationaler Akteur, der als „Schmied seines eigenen Glücks" auch die Wirkungsweise von Institutionen verändert. Bewertet man die Rolle der Regionalplanung aus dieser Perspektive, sollte sie durch Verzahnung mit Strategien der regionalen Wirtschaftsförderung darauf abzielen, ein optimales Zusammenspiel verschiedener Flächennutzungen zu erreichen, das wirtschaftliche Entfaltungsmöglichkeiten erlaubt und den Ansprüchen an einen attraktiven Lebensraum gerecht wird. 
(2) In der Metropole Ruhr sollte das Subsidiaritätsprinzip gelten, d.h. die regionale Planung und Wirtschaftsförderung ist vor allem eine "Klammer", die für die erforderliche Koordination innerhalb des Siedlungsraums sorgt und dessen Gesamtgewicht im europäischen bzw. weltweiten Standortwettbewerb stärkt.

(3) Konkurrenz belebt das Geschäft. Aus regionalökonomischer Perspektive ist zu befürworten, dass die Kommunen des Ruhrgebiets durchaus auch weiterhin untereinander im Wettbewerb stehen, lokalspezifische Standortvorteile optimal zu fördern. Diese lokalen Besonderheiten sollten sich zu einem umfassenden Standortportfolio ergänzen, das insgesamt attraktiver ist als das Angebot einzelner Städte bzw. Ballungsräume.

Das grundlegende Problem, das aus ökonomischer Sicht durch Veränderungen wie die wirtschaftliche Globalisierung und den demographischen Wandel ausgelöst wird, sind die unzureichenden Möglichkeiten des Einzelnen, sich an diese neuen Gegebenheiten durch ein verändertes ökonomisches Verhalten anzupassen. Diese Einschränkung wird häufig durch die starre Struktur der Institutionen ausgelöst, die das Handeln von Individuen und Unternehmen als Rahmen begleiten. Darüber hinaus kann eine Wirtschaftspolitik, die Wandlungsprozesse aktiv gestalten möchte, statt sich darauf zu beschränken, den individuellen Entscheidungsträgern die besten Rahmenbedingungen für ihre selbstständige und eigenverantwortliche Gestaltung zu schaffen, ebenfalls negative Effekte auslösen. Angesichts der begrenzten Möglichkeiten staatlicher Planung effizienten Wirtschaftens ist bei dieser aktiven Gestaltung Zurückhaltung dringend anzuraten.

Auch wenn mittlerweile in vielen Politikbereichen, etwa der aktiven Arbeitsmarktpolitik oder der Technologiepolitik, gewisse Erkenntnisfortschritte zu verzeichnen sind: Eine zielführende Wirtschaftspolitik beseitigt die Hemmnisse starrer Strukturen, ohne sich anzumaßen, „das Heft selber in die Hand zu nehmen“. Welche staatliche Maßnahme überhaupt im vorgesehenen Sinne wirkt und welche man unterlassen sollte, bleibt weitgehend der künftigen Forschung vorbehalten. Über die Wirkungen regionalpolitischer Einflussnahmen auf die Prosperität von Unternehmen und Individuen ist bislang zwar Etliches bekannt, aber längst nicht genug. Die Erwartungshaltung an die möglichen ökonomischen Effekte der Regionalplanung für die „Metropole Ruhr“ sollte daher in Grenzen gehalten werden.
Die Autoren danken Anette Hermanowski, Marlies Tepaß und Joachim Schmidt für die Unterstützung bei der Durchführung des Projekts. - Die vorliegende Arbeit basiert auf einem Beitrag des RWI Essen zu einer Anhörung des Landtags NordrheinWestfalen (RWI Essen 2007).

\section{Anmerkungen}

(1) Als "Ruhrgebiet" wird im Folgenden das in Abbildung 1 dargestellte RVR-Verbandsgebiet verstanden. Die in diesem Beitrag verwendete Bezeichnung "Metropole Ruhr" bezieht sich auf dieses Gebiet.

(2)

Nach dem Gesetz zur Übertragung der Regionalplanung für die Metropole Ruhr auf den Regionalverband Ruhr (Landesplanungsgesetz NRW vom 3. Mai 2005, GV. NRW. S. 430, geändert durch Gesetz vom 24. Mai 2007) wird der RVR am Tag der Bekanntmachung des Ergebnisses der Kommunalwahl 2009 regionaler Planungsträger für das Verbandsgebiet.

(3)

Einen Einstieg in die wirtschaftswissenschaftliche Literatur zu Fragen der Standortwahl von Unternehmen, Haushalten und Verwaltungseinheiten bieten Nijkamp/Mills (1986).

(4)

Vgl. auch frühere Stellungnahmen der Autoren zur Ruhrgebietsthematik in Schmidt 2005, RWI Essen 2006, Lageman et al. 2005, 2006

(5)

Hierauf zielte der die Thematik etwas zuspitzende Titel der Studie ab: „Gibt es noch ein Ruhrgebiet?"( Schrumpf et al. 2001).

(6)

Befragt wurden von 16 Interviewern in 20 bis 30 -minütigen „faceto-face-Gesprächen" 1012 Personen (zur Methodik vgl. Stuke/ Kruck 2002, S. 9).

(7)

Die zu einer "Ringstadt“ zusammen gewachsene Agglomeration der Großstädte Amsterdam, Den Haag, Rotterdam und Utrecht, die Teile von fünf Provinzen umfasst und rd. 7 Mill. Einwohner zählt

(8)

Die Kooperation kommunaler Behörden oder gar die administrative Integration von Kommunen stellt sich auch in anderen Ländern höchst komplex und ambivalent dar, so z.B. auch im US-Bundesstaat Illinois (Gordon 2007).

(9)

Zu den allgemeinen Aufgaben der Regionalplanung vgl. Spitzer 1995 


\section{Literatur}

Adam, B. (2006): Europäische Metropolregionen in Deutschland: Perspektiven für das nächste Jahrzehnt. Sankt Augustin. = KASPublikationen Kommunalpolitik, 34.

Blotevogel, H.H. (2001): Industrielle Kulturlandschaft im Ruhrgebiet. Die Geschichte einer schwierigen Annäherung. Duisburg. $=$ Diskussionspapier 3/2001 des Institut für Geographie der Gerhard-Mercator-Universität Duisburg.

Blotevogel, H.H. (2006): Metropolregion Rhein-Ruhr. In: Geogr. Rundschau 58, H. 1, S. 28-36.

BMVBS - Bundesministerium für Verkehr, Bau und Stadtentwicklung (2006): Leitbilder und Handlungsstrategien für die Raumentwicklung in Deutschland. Verabschiedet von der Ministerkonferenz für Raumordnung am 30. Juni 2006. Berlin.

Camagni, R. (2002): On the concept of territorial competitiveness: sound or misleading? In: Urban Studies 39, H. 13, S. 2395-2411.

Davy, B. (2004): Die neunte Stadt. Wilde Grenzen und Städteregion Ruhr 2030. Wuppertal.

Ebert, R. ; Gnad, F; Van Ooy, U.; Kunzmann, K.R. (2005): Wandel durch Kultur(wirtschaft) im Ruhrgebiet. Kultur(wirtschaft) durch Wandel. Ein Beitrag zur Bewerbung "Essen für das Ruhrgebiet - Kulturhauptstadt Europas 2010“. Hrsg. Ministerium für Wirtschaft, Mittelstand und Energie des Landes Nordrhein-Westfalen, Regionalverband Ruhrgebiet. Düsseldorf, Essen.

Florida, R. (2005): Cities and the creative class. London, New York.

Fujita, M.; Krugman, P.R.; Venables, A.J. (2001): The Spatial Economy. Cities, Regions and International Trade. Boston, London.

Fürst, D. (2001): Regional Governance - ein neues Paradigma der Regionalwissenschaften? In: Raumforsch. u. Raumordnung 59, H. 5/6, S. 370-380.

Gordon, V. (2007): Partners or Competitors? Perceptions of Regional Economic Development Cooperation in Illinois. In: Economic Development Quarterly 21, H. 1, S. 60-78. http://edq. sagepub.com/cgi (content/abstract/21/1/60).

Hirschmann, A.O. (1958): The strategy of economic development. New Haven.

Kilper, H. (1995): Von regionaler Selbstregulierung zu interregionaler Konkurrenz. Wandel kommunaler Zusammenarbeit im Ruhrgebiet. In: Kommunalverband Ruhrgebiet (Hrsg.): Kommunalverband - Ruhrgebiet. Wege, Spuren. Festschrift zum 75jährigen Bestehen des Kommunalverbands Ruhrgebiet. Essen, S. $69-103$.

Krugman, P.R. (1991): Geography and trade. Cambridge, Mass.

Kühn, S. (1996): Der Regionalverband Ruhrgebiet als regionale Interessenvertretung. In: Bovermann, R; Goch, S.; Priamus, H.-J. Hrsg.): Das Ruhrgebiet - Ein starkes Stück Nordrhein-Westfalen. Politik in der Region 1946-1996. Essen. = Schriftenreihe des Instituts für Stadtgeschichte, Beiträge, Bd. 7 .

Lageman, B. et al. (2005): Strukturwandel ohne Ende? Aktuelle Vorschläge zur Revitalisierung des Ruhrgebiets und ihre Bewertung. Essen. = RWI-Materialien, H. 20.
Lageman, B.; Neumann, U.; Schmidt, C.M. (2006): Und täglich grüßt die Subvention - wie kann die erfolgreiche Revitalisierung des Ruhrgebiets gelingen? In: Z. f. Wirtschaftsgeographie 50, H. 3/4, S. 232-244.

LDS NRW - Landesamt für Datenverarbeitung und Statistik Nordrhein-Westfalen (2002): Pendlerrechnung Nordrhein-Westfalen 2002. CD-ROM. Düsseldorf.

Maier, G.; Tödtling, F. (2002): Regional- und Stadtökonomik, Bd. 2: Regionalentwicklung und Regionalpolitik. 2. Aufl., Wien, New York.

Malecki, E.J. (1991): Technology and economic development. - New York 1991

Martin, R.; Sunley, P. (2006): Path dependence and regional economic evolution. In: Journ. of Economic Geography 6, H. 4, S. 395-437.

Myrdal, G. (1957): Economic theory and underdeveloped regions. London.

Neumann, U.; Schatz-Bergfeld, M. (1999): Meinungsbilder und Verhaltensmuster in Duisburg-Marxloh. Entwicklung 1997 bis 1999 und Zusammenhang mit der Strukturentwicklung des Stadtteils. Hrsg. N.U.R.E.C.-Institute. Duisburg. = Monitoring kleinräumiger Entwicklungsprozesse, H. 6.

Neumann, U.; Tonks, R. (2007): The infrastructure of a leading European logistics metropolis. Duisburg, Essen et al. = EUROPOLIS N.U.R.E.C. Working Papers, H. 9.

Nijkamp, P; Mills, E.S. (1986): Advances in Regional Economics. In: Nijkamp, P. (Hrsg.): Handbook of Regional and Urban Economics, Bd. 1. Amsterdam, S. 1-17.

Pankoke, E. (1990): Öffentliche Verwaltung 1918-1975. In: Köllmann, W. et al. (Hrsg.): Das Ruhrgebiet im Industriezeitalter. Bd. 2: Geschichte und Entwicklung. Düsseldorf, S. 7-66.

Peck, J. (2003): Doing Regulation. In: The Oxford Handbook of Economic Geography. Oxford, New York u. a. S. 66-80.

Perroux, F.(1950): Economic spaces: theory and application. In: Quarterly Journ. of Economics 64, S. 90-97.

Porter, M.E. (1990): The Competitive Advantage of Nations. New York.

Porter, M.E. (1998): Clusters and the New Economics of Competition. In: Harvard Business Review, November-December, S. $77-90$.

Romer, P.M. (1990): Endogenous technological change. In: Journ. of Political Economy 98, S. 71-102.

RVR-Gesetz (2007): Gesetz über den Regionalverband Ruhr in der Fassung der Bekanntmachung vom 3. Februar 2004 (GV NRW S. 96), geändert durch Gesetz vom 16. Nov. 2004 /GV.NRW S. 644), geändert durch Gesetz vom 5. April 2005 (GV.NRW S. 351), geändert durch Gesetz vom 5. Juni 2007 (GV NRW S. 212). Eigendruck des Regionalverbandes Ruhr. Essen.

RWI Essen (2006): Strukturförderpolitik für Nordrhein-Westfalen. Stellungnahme zur öffentlichen Anhörung des Hauptausschusses des Landtags Nordrhein-Westfalen zum Antrag der Fraktion der SPD „Strukturförderpolitik für NRW 2007-2013 - Den Strukturwandel weiter erfolgreich gestalten" am 9. Februar 2006. Essen. $=$ RWI-Projektberichte. 
RWI Essen (2007): Gesetz zur Übertragung der Regionalplanung für die Metropole Ruhr auf den Regionalverband Ruhr. Stellungnahme zur Anhörung von Sachverständigen gemäß $\$ 56$ der Geschäftsordnung des Ausschusses für Wirtschaft, Mittelstand und Energie des Landtags Nordrhein-Westfalen am 14. März 2007. Essen. = RWI-Projektberichte.

Schmidt, C.M. (2005): Champions League oder Provinztheater? - Chancen des Ruhrgebiets im Wettbewerb der Regionen. In: Nationalbank AG (Hrsg.): Geschäftsbericht 2004: An Rhein und Ruhr. Essen, S. 98-113.

Schrumpf, H.; Budde, R.; Urfei, G. (2001): Gibt es noch ein Ruhrgebiet? Essen. = Schriften u. Materialien zur Regionalforschung, Bd. 6.

Spethmann, H. (1933, 1995): Das Ruhrgebiet im Wechselspiel von Land und Leuten, Wirtschaft, Technik und Verkehr. Zweiter Band: Die Entwicklung zum Großrevier seit Mitte des 19. Jahrhunderts. Essen (1933, unveränderter Nachdruck 1995)

Spitzer, H. (1995): Einführung in die räumliche Planung. Stuttgart.

Steinacher, B. (2006): Aktivitäten und Vernetzung der Metropolregionen in Deutschland und Europa. Rede von Dr. Bernd Steinacher, Regionaldirektor Verband Region Stuttgart, Sprecher Initiativkreis Europäische Metropolregionen in Deutschland, im Rahmen der Tagung METROPOLREGIONEN - Bausteine für ein wettbewerbsfähiges Deutschland in Europa während des Kongresses euregia am 24. Oktober 2006 in Leipzig. Stuttgart
Stuke, F.R.; Kruck, P. (2002): Die Große Ruhrgebiet-Umfrage BIFAK - Bochumer Institut für angewandte Kommunikationsforschung. Bochum.

Technische Universität Berlin, Fakultät VII - Architektur Umwelt Gesellschaft (2003): Modellvorhaben Regionen der Zukunft, Initiativkreis Metropolregionen in Deutschland: Strategiepapier Berlin.

Voß, A. (2007): Wie real ist die Ruhrstadt - neue Umsetzungschancen einer alten Vision. Essen. www.initiative-ruhrstadt.de.

Willamowski, G. (2001): Ruhrstadt - eine Vision zeigt den Weg Die Region braucht regionale Handlungsstrategien. In: Kommunalverband Ruhrgebiet (Hrsg.): Standorte. Jahrbuch Ruhrgebiet 2001/2002. Essen, S. 26-30.

Zöpel, C. (2005): Weltstadt Ruhr. Essen.

Dr. Bernhard Lageman

Dr. Uwe Neumann

Prof. Dr. Christoph M. Schmidt, Ph. D.

RWI Essen

(Rheinisch-Westfälisches Institut

für Wirtschaftsforschung)

Hohenzollernstraße 1-3

45128 Essen

E-Mail: neumann@rwi-essen.de 